ISSN: 2638-6062

\title{
Alcohol in Mexico: Dying, Like Sucking, Also Is Gerundio
}

\author{
Gabriel Miranda Nava* \\ Department of Neurology, Hospital Center, Mexico, USA
}

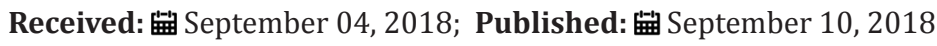

*Corresponding author: Gabriel Miranda Nava, Chief of Neurology, Hospital Center of the Presidential General Staff, Mexico, USA

\section{Opinion}

As I commented previously, alcoholism is by far the addiction with the highest prevalence and incidence in our country; only in 2011 were detected around six million people with a harmful consumption of alcohol, and that they developed some of the 52 diverse conditions already described. And it is not only the liver, with its sequence of intoxication, congestion, hepatic steatosis (some call it fatty liver), hepatitis, cirrhosis and some even liver cancer; we also have alcoholic pancreatitis, heart disease (alcoholic cardiomyopathy), mental problems such as Wernicke's disease or Korsakoff's psychosis, chronic depression very resistant to drugs, as well as less frequent Marchiafava-Bignami syndrome, polyneuropathies (the second most frequent being alcoholic). in our country), myopathies, haematological consequences with lower production of leukocytes and greater propensity to various infections such as tuberculosis or various pneumonias; Gastrointestinal complications such as gastritis, irritable bowel syndrome, esophagitis and a significant increase in the risk of gastric cancer.

Tired, right? And the general hospitals are full of patients with the afore mentioned illnesses, and with the family that suffers the consequences of a fatal mental dynamic (who can tolerate an alcoholic at home?) Or the catastrophic economic cost since everything went in " the suck "for years. And the worst is yet to come, because now it is observed, according to the ENA (National Survey of Addictions) that already $50 \%$ of students over 15 years of age already tested it, and the liver cirrhosis line increased by $50 \mathrm{At}$ 40 years of age, these are, every day we see more young people with greater complications and who move to the extreme poverty line because of the expense derived from their pocket in medical care. How to realize a person who takes compulsively and harmfully? We always think that the chronic alcoholic is that "teporochito" of the street, pulled, smelly and rejected by society, but that is the big lie, since these street characters only correspond to $3 \%$ of the population that suffers from alcoholism or other addictions; our large number of patients are weekend drinkers, many are "social drinkers" or "I only drink every fortnight" but that regularity worthy of a Swiss watch is the most distinctive characteristic of the addict in process, "the belly "of the sick population, but accepted, or socially denied. The following signs or behaviors are suspected of having alcoholism:

a) We begin with the antecedent of a direct family member who is or was a compulsive drinker, since this factor alone increases to 4 times the risk of presenting a harmful behavior with alcohol; It is difficult to think of another mental illness with so much family dominance.

b) Uncontrollable desire to continue consuming alcohol, with the loss associated with the control of it.

c) Need to drink alcohol "to feel good".

d) Increasing amounts of alcohol are increasingly needed.

e) Present symptoms typical of withdrawal syndrome (sweating, despair, anxiety, insomnia).

f) Other pleasurable activities have been abandoned because of alcohol consumption.

g) The individual loses consciousness of the harm of alcohol on himself.

h) Experience frequent forgetting.

i) Be a lone drinker or do it in a hidden way.

j) Use many monetary resources to meet only the goal of drinking, it affects the household economy and even if solid situations such as the tuition of children, scheduled trips or parties are lost, there is no change in behavior. 
k) To be understood, it is more important to drink than everything else, there is nothing else, the world can explode and yet you can continue drinking.

l) This is why it is not necessary for someone to reach very advanced stages to act, and we must remember that the

To Submit Your Article Click Here: Submit Article

DOI: $10.32474 /$ PRJFGS.2018.02.000134

alcoholic is confronted, told things in advance, not spoiled, and put in situations of final decision, such as "or the alcohol or me. "Let's avoid this disease, since as we say, both dying and sucking, it is also gerund.

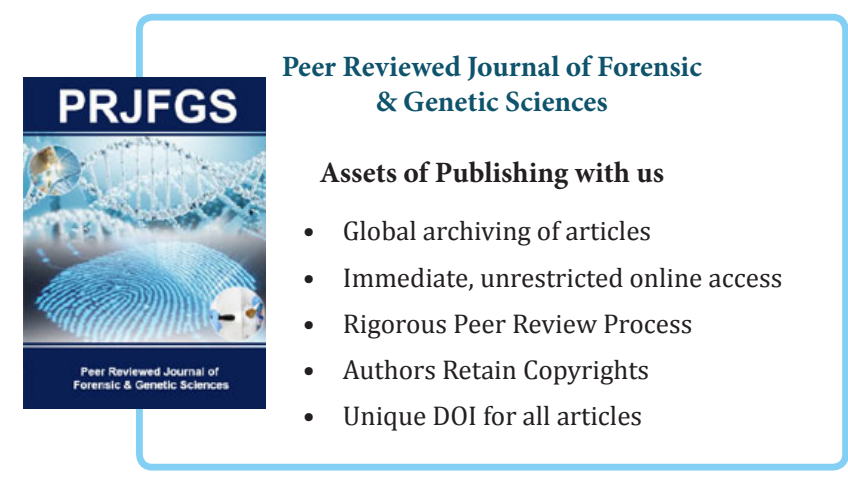

